\title{
Efeito do Exercício de Ultrarresistência Sobre Parâmetros de Estresse Oxidativo
}

\author{
Effect of the Ultra-endurance Exercise on Oxidative \\ Stress Parameters
}

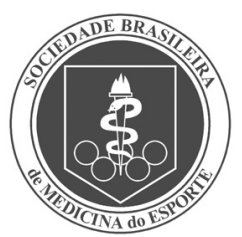

Artigo Original
Cláudia Dornelles Schneider ${ }^{1}$ Márcio Martins Silveira²,

José Cláudio Fonseca Moreira ${ }^{3}$, Adriane Belló-Klein ${ }^{4}$,

Alvaro Reischak de Oliveira ${ }^{5}$

1. Laboratório de Pesquisa do Exercício (Lapex) - Escola de Educação Física - UFRGS - Porto Alegre, RS.

2. Centro de Estudo em Estresse Oxidativo - Departamento de Bioquímica - Instituto de Ciências Básicas da Saúde - UFRGS - Porto Alegre, RS.

3. Centro de Estudo em Estresse Oxidativo - Departamento de Bioquímica - Instituto de Ciências Básicas da Saúde - UFRGS - Porto Alegre, RS

4. Laboratório de Espécies Ativas de Oxigênio - Departamento de Fisiologia - Instituto de Ciências Básicas da Saúde - UFRGS - Porto Alegre, RS.

5. Laboratório de Pesquisa do Exercício (LAPEX) - Escola de Educação Física - UFRGS - Porto Alegre, RS.

Endereço para correspondência: Alvaro Reischak de Oliveira. Rua Felizardo, 750 - Bairro Jardim Botânico - 90690-200 - Porto Alegre, RS. Tel.: (51) 3308-5817 / fax: (51) 3308-5842

E-mail: aroliveira@esef.ufrgs.br

Submetido em 21/05/2007

Versão final recebida em 18/04/2008 Aceito em 24/10/2008

\begin{abstract}
RESUMO
Introdução: Exercícios de longa duração podem levar ao desequilíbrio entre os sistemas pró e antioxidante, acarretando dano a lipídeos, proteínas e DNA. Entretanto, alguns estudos avaliando triatlo Ironman observaram proteção aos lipídeos. Objetivo: Avaliar parâmetros de estresse oxidativo após uma competição de meio Ironman. Métodos: Participaram 11 sujeitos com idade de 31,1 $\pm 3,3$ anos, massa corporal de 72,4 \pm $5,4 \mathrm{~kg}$, estatura de $176,2 \pm 4,8 \mathrm{~cm}$, gordura corporal de $9,8 \pm 3,3 \%, \dot{V}_{2 \text { máx }}$ na corrida de $60,7 \pm 6,0 \mathrm{~mL} / \mathrm{kg} / \mathrm{min}$. Foram mensurados: dano a lipídeos através da quimiluminescência nos eritrócitos e TBARS no plasma, dano a proteínas através das carbonilas plasmáticas, ácido úrico e compostos fenólicos plasmáticos, assim como a atividade antioxidante enzimática da catalase, superóxido dismutase e glutationa peroxidase nos eritrócitos. Resultados: Houve redução na atividade da enzima superóxido dismutase (23,24 $\pm 1,49$ para 20,77 $\pm 2,69 \mathrm{U}$ SOD/mg proteína, $p=0,045)$, e aumento no ácido úrico (40,81 \pm 10,68 para 60,33 $\pm 6,71 \mathrm{mg} / \mathrm{L}, \mathrm{p}<0,001)$ logo após a competição. Não houve diferença estatisticamente significativa na atividade das enzimas antioxidantes catalase e glutationa peroxidase e nos compostos fenólicos totais, assim como não foi observado dano a lipídeos (TBARS e quimiluminescência) e proteínas (carbonilas). Conclusão: Esse grupo de atletas não sofreu estresse oxidativo, provavelmente devido à liberação de ácido úrico e outros antioxidantes no plasma.
\end{abstract}

Palavras-chave: radicais livres, ácido úrico, superóxido dismutase.

\begin{abstract}
Ultra-endurance exercises can cause imbalance between the pro and antioxidant systems, leading to lipid, protein and DNA damage. Nevertheless, some studies evaluating Ironman triathlon found protection to lipids. Purpose: To evaluate oxidative stress parameters after a half-Ironman competition. Methods: Eleven subjects aged $31.1 \pm 3.3 \mathrm{yr}$, body weight $72.4 \pm 5.4 \mathrm{~kg}$, height $176.2 \pm 4.8 \mathrm{~cm}$, body fat $9.8 \pm 3.3 \%$, $\mathrm{VO}_{2 \text { máx }}$ on run $60.7 \pm 6.0 \mathrm{~mL} / \mathrm{kg} / \mathrm{min}$, participated in this study. The following data were measured: lipid damage by chemoluminescence in erythrocyte and TBARS in plasma, protein damage by plasmatic carbonyls, uric acid and phenolic compounds, as well as the antioxidant enzymatic activity of catalase, superoxide dismutase and glutathione peroxidase in erythrocytes. Results: Reduction in superoxide dismutase (23.24 \pm 1.49 to $20.77 \pm$ $2.69 \mathrm{U} \mathrm{SOD} / \mathrm{mg}$ protein, $p=0.045)$, and increase in uric acid (40.81 \pm 10.68 to $60.33 \pm 6.71 \mathrm{mg} / \mathrm{L}, \mathrm{p}<0.001$ ) were found immediately after the competition. No statistically significant differences were found in catalase and glutathione peroxidase or phenolic compounds. Lipid (TBARS and chemoluminescence) or protein (carbonyl) damage was not observed either. Conclusion: These athletes did not suffer oxidative stress, probably due to uric acid and other plasmatic antioxidants release in the plasma.
\end{abstract}

Keywords: free radicals, uric acid, superoxide dismutase.

\section{INTRODUÇÃO}

Exercícios extenuantes ou de longa duração estão relacionados ao dano tecidual e podem causar estresse oxidativo, uma vez que o exercício aumenta o consumo de oxigênio e causa um distúrbio na homeostase pró e antioxidante intracelular ${ }^{(1)}$. O treinamento físico pode prevenir parcialmente a formação de radicais livres (RL) no exercício exaustivo ${ }^{(2)}$ e aumentar as defesas antioxidantes $(\mathrm{AO})^{(3)}$.

Ainda não foi descoberto nenhum índice absoluto e definitivo de estresse oxidativo (EO); no entanto, os RL, ao reagir com compostos biológicos, produzem derivados oxidados, cujos níveis podem ser usados como índices de EO. Podem-se medir a peroxidação lipídica, o dano a proteínas e DNA, e mudanças no estado dos compostos AO e atividade de enzimas AO. Tem crescido o interesse por esportes de longa duração como maratonas, corridas de aventura e triatlo de longa distância como Ironman e meio Ironman. Artigos relacionando exercício de longa duração ao EO apontam para o desequilíbrio entre os sistemas pró-oxidante e antioxidante, podendo acarretar dano a lipídeos ${ }^{(4)}$ e proteínas $^{(5)}$ de membrana, assim como ao DNA ${ }^{(6)}$. Entretanto, estudos avaliando triatletas após o Ironman do Havaí observaram proteção aos lipídeos de membrana ${ }^{(7)}$. Além disso, estudos mais recentes investigam se a suplementação AO seria capaz de proteger do dano oxidativo em Ironman, mas os estudos ainda não são concordantes ${ }^{(8,9)}$. Como pudemos observar, os resultados do efeito do exercício de longa duração sobre o EO em humanos são conflitantes. Não foi possível encontrar artigos que apresentassem a medida desses parâmetros em atletas brasileiros. Sendo assim, o objetivo deste estudo foi mensurar os parâmetros bioquímicos sanguíneos de estresse oxidativo em triatletas brasileiros após uma competição de meio Ironman. 


\section{MÉTODOS}

Participaram deste estudo 13 triatletas voluntários do sexo masculino, residentes na cidade de Porto Alegre e Grande Porto Alegre. A amostragem foi intencional. Os sujeitos participaram de uma competição de meio Ironman caracterizada por 1,9km de natação, $90 \mathrm{~km}$ de ciclismo e 21 km de corrida, integrante do calendário da Federação Gaúcha de Triatlo, que ocorreu em 15/2/2004 no Balneário Pinhal, RS. Todos os atletas estavam engajados em um treinamento que objetivava a participação no Ironman de Florianópolis.

Dos 13 atletas que realizaram a coleta de sangue inicial, dois não completaram a etapa do ciclismo, sendo automaticamente excluídos do estudo. Dessa forma, a amostra final foi composta por 11 triatletas. Os indivíduos foram informados do objetivo, desconfortos, riscos e benefícios do estudo e assinaram um termo de consentimento livre e esclarecido, de acordo com a declaração de Helsinque. O estudo foi aceito pelo comitê de ética em pesquisa da UFRGS.

Os atletas foram orientados a ingerir seu desjejum habitual, assim como a manter os procedimentos de alimentação e hidratação a que estavam acostumados no dia da competição, não havendo interferência dos pesquisadores nesses parâmetros. Foram coletadas amostras sanguíneas antes e logo após a competição por um profissional habilitado. As amostras de sangue foram preparadas e aliquotadas em tubos plásticos de 1,5mL, congeladas e transportadas em um contêiner contendo nitrogênio líquido para o laboratório, onde foram armazenadas a $-73^{\circ} \mathrm{C}$ para posteriores análises.

Previamente à competição, os atletas foram avaliados no laboratório de pesquisa do exercício da Escola de Educação Física da UFRGS. Para medir o consumo máximo de oxigênio na esteira rolante, foi realizado um teste incremental em rampa, utilizando um sistema automático ergoespirométrico, respiração a respiração (CPX/d, Medical Graphics Corporation; St Paul, MN). Para medir a massa corporal foi utilizada balança digital, com resolução de $100 \mathrm{~g}$ e capacidade máxima de 180,0kg, e para medir a estatura foi utilizado o estadiômetro com precisão de 0,5cm e capacidade máxima de $210 \mathrm{~cm}$, ambos da marca Urano (Canoas, RS). Para avaliar o percentual de gordura corporal foi utilizado o método de dobras cutâneas, utilizando o adipômetro Lange, com a equação de predição de Jackson e Pollock (1978) com sete dobras cutâneas para atletas.

Análises bioquímicas: Uma amostra de $7 \mathrm{~mL}$ de sangue foi coletada de uma veia na região antecubital e armazenada em tubos plásticos com 100 $\mu \mathrm{L}$ de EDTA a 10\%. O sangue foi centrifugado por 5 min a $1.000 \mathrm{~g}$ em centrífuga refrigerada, o plasma aliquotado e congelado para análise das carbonilas, TBARS, compostos fenólicos e ácido úrico. Os eritrócitos foram lavados três vezes com igual volume de solução fisiológica e mantidos refrigerados. Uma parte foi preparada e congelada para posterior análise da atividade de enzimas antioxidantes e outra parte utilizada para a análise da quimiluminescência (QL), realizada no dia seguinte ao da coleta de sangue. Foram utilizados os seguintes equipamentos: (a) para análise da QL: um contador ( LKB Wallac Rack Beta Liquid Scintillation Counter - 1209, LKB Producter AB, Bromma, Suécia), com o circuito de coincidência desconectado, utilizando o canal de trítio; (b) para mensurar as carbonilas e a atividade das enzimas catalase, superóxido dismutase e glutationa peroxidase foi utilizado um espectrofotômetro (UV-Visible Spectrophotometer - Cary 1E, Varian, Austrália); (c) para medir o TBARS, ácido úrico e compostos fenólicos foi utilizado um espectrofotômetro (Spectronic 20 Genisys Spectrophotometer, Spectronic Instruments, NY, EUA).

Atividade das enzimas antioxidantes: Superóxido dismutase (SOD): foi determinada nos eritrócitos pela taxa de inibição da auto-oxidação do pirogalol a 420nm, a partir de uma curva padrão de SOD disponível comercialmente. Meio de reação: 50 mmol/L de tampão tris (pH 8,2), $24 \mathrm{mmol} / \mathrm{L}$ pirogalol e $30 \mu \mathrm{mol} / \mathrm{L}$ catalase. Resultados expressos em $\mathrm{U}$ SOD/mg proteína ${ }^{(10)}$. Catalase (CAT): foi medida nos eritrócitos pelo consumo de $\mathrm{H}_{2} \mathrm{O}_{2}$ a $240 \mathrm{~nm}$. Meio de reação: $50 \mathrm{mmol} / \mathrm{L}$ de tampão fosfato $\mathrm{pH} 7,4$ e 0,3mol/L de $\mathrm{H}_{2} \mathrm{O}_{2}$. Resultados expressos em $\mathrm{pmol} / \mathrm{mg}$ proteína(11). Glutationa peroxidase (GPX): Foi medida através da oxidação do NADPH a 340nm. Meio de reação: $143 \mathrm{mmol} / \mathrm{L}$ tampão fosfato $\mathrm{pH} 7,5,1 \mathrm{mmol} / \mathrm{L}$ azida sódica, 0,5 mmol/L hidroperóxido de tert butil, 0,25U/mL glutationa redutase, 0,24mmol/L NADPH e $5 \mathrm{mmol} / \mathrm{L}$ glutationa reduzida. Resultados expressos em mmol/L/min/mg proteína ${ }^{(12)}$. QL: foi mensurada nos eritrócitos usando 400 mmol/L de hidroperóxido de tert butil. A luz emitida é medida em um contador de cintilação, adaptado para contar a emissão de luz usando um canal de trítio ( $L K B$ Rack Beta Liquid Scintillation Spectrometer, modelo 1215, LKB-Produkter

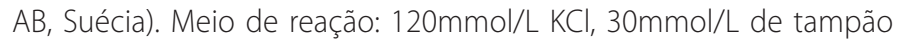
fosfato $\mathrm{pH} 7,4$. Resultados expressos em cps/mg Hb ${ }^{(13,14)}$. Substâncias reativas ao ácido tiobarbitúrico (TBARS): foi mensurado no plasma. Juntar $300 \mu$ l de plasma com $600 \mu$ l de TCA (2:1), centrifugar a $10.000 \mathrm{~g}$ por 10 min. Reagir $500 \mu \mathrm{l}$ do sobrenadante com 500 $\mu$ l de TBA (0,67\%). Ferver 20 minutos e após resfriar $5 \mathrm{~min}$. Ler a 532nm. Resultados expressos em pmol/mg proteína ${ }^{(15)}$. Ácido úrico: Foi analisado no plasma através do kit comercial Uricostat enzimático AA (Wiener Laboratórios - Argentina). Resultados expressos em mg/L. Carbonilas: o método consiste na reação de dinitrofenilhidrazina (DNPH) com as carbonilas das proteínas formando hidrazonas, que são medidas espectrofotometricamente a $360 \mathrm{~nm}$. (i) coloca-se o DNPH com o plasma. Para cada amostra, utiliza-se outro tubo, onde mede-se a proteína total da amostra, usando $\mathrm{HCl}$ ao invés de DNPH; (ii) é adicionado o TCA a 20\% e em seguida TCA a 0\% em ambos os tubos, para a precipitação das carbonilas e proteínas; (iii) o precipitado é lavado com etanol e acetato de etila para remover o DNPH livre e lipídios contaminantes. O precipitado final é dissolvido em guanidina e lido a 360nm. A proteína total da amostra lê-se a 280nm. Resultados expressos em nM/mg proteína ${ }^{(16)}$. Compostos fenólicos totais: foram dosados no plasma utilizando o método modificado do Folin-Ciocalteau. 100 $\mu \mathrm{L}$ de plasma foram misturados a 300 $\mu \mathrm{L}$ de ácido tricloroacético a 15\%, e centrifugados a $10.000 \mathrm{~g}$ por $10 \mathrm{~min}$ a $4^{\circ} \mathrm{C}$. Após,

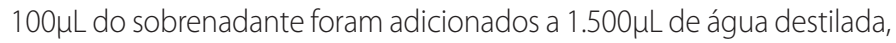
$100 \mu \mathrm{L}$ Folin-Ciocalteau e $200 \mu \mathrm{L}$ de solução saturada de $\mathrm{Na}_{2} \mathrm{CO}_{3}$. As amostras foram então comparadas espectrofotometricamente a $725 \mathrm{~nm}$ com amostras de ácido tânico, que serviu como padrão. Resultados expressos em equivalentes de ácido tânico/mL plasma ${ }^{(17)}$. Medida da proteína: foi medida de acordo com o método amplamente utilizado de Lowry et al. ${ }^{(18)}$.

Tratamento estatístico: Foi utilizado o teste de Shapiro-Wilk para testar a normalidade dos dados. A comparação entre antes e depois da competição foi realizada utilizando o teste $t$ pareado, visto que os dados apresentaram distribuição normal. Os resultados foram expressos em média \pm desvio padrão (DP) e foram analisados com o auxílio do pacote estatístico Statistical Package for the Social Sciences (SPSS, 10.0). O nível de significância aceito foi de 5\%.

\section{RESULTADOS}

As características descritivas da amostra compreendem idade de $31,1 \pm 3,3$ anos, massa corporal de 72,4 \pm 5,4kg, estatura de 176,2 \pm $4,8 \mathrm{~cm}$, gordura corporal de 9,8 $\pm 3,3 \%$, e $\dot{\mathrm{V}} \mathrm{O}_{2 \text { máx }}$ na corrida $60,7 \pm$ $6,0 \mathrm{~mL} / \mathrm{kg} / \mathrm{min}$.

Os atletas participaram nas categorias elite, 25-29 anos e 30-34 anos, classificando-se até o oitavo lugar dentro de suas respectivas categorias. Levaram entre 4h27min51s e 5h30min23s (300,2 $\pm 24,3$ min) para completar a competição. 
A tabela 1 apresenta os parâmetros de estresse oxidativo antes e após a competição de meio Ironman.

Tabela 1. Parâmetros bioquímicos de estresse oxidativo em uma competição de meio Ironman

\begin{tabular}{l|c|c|c}
\hline \multicolumn{1}{c|}{ Variáveis $(\mathbf{n}=\mathbf{1 1})$} & Antes & Depois & $\mathbf{p}$ \\
\hline Quimiluminescência (cps/mg Hb) & $16.249 \pm 1.577$ & $16.989 \pm 1.532$ & 0,331 \\
\hline TBARS (pmol/mg prot) & $5,66 \pm 2,26$ & $7,33 \pm 1,55$ & 0,068 \\
\hline Carbonilas (nmol/mg prot) & $5,43 \pm 1,64$ & $5,10 \pm 1,47$ & 0,582 \\
\hline Fenólicos (equival. ácido tânico/mL plasma) & $87,7 \pm 19,2$ & $101,4 \pm 21,4$ & 0,080 \\
\hline Ác. úrico (mg/L) & $40,8 \pm 10,7$ & $60,3 \pm 6,7$ & $0,000^{*}$ \\
\hline Catalase (pmol/mg prot) & $21,4 \pm 4,0$ & $18,4 \pm 3,3$ & 0,058 \\
\hline Superóxido dismutase (U SOD/mg prot) & $23,3 \pm 1,4$ & $21,0 \pm 2,7$ & $0,044^{*}$ \\
\hline Glutationa peroxidase (mmol/min/mg prot) & $4,19 \pm 0,51$ & $3,95 \pm 0,66$ & 0,239
\end{tabular}

Resultados descritos como média \pm desvio padrão. Comparação por teste $t$ pareado.

* Diferença significativa entre antes e depois $(p<0,05)$.

Foi constatado que após a competição de meio Ironman houve aumento significativo de aproximadamente 48\% nas concentrações plasmáticas de ácido úrico ( $p<0,001)$ e redução significativa de aproximadamente 10\% na atividade da enzima superóxido dismutase $(p=0,044)$.

Entretanto, não observamos diferença estatisticamente significativa para os parâmetros de dano a lipídeos, avaliados pelas técnicas do TBARS e quimiluminescência, ou dano às proteínas, avaliado pela técnica das carbonilas.

A atividade das enzimas antioxidantes catalase e glutationa peroxidase assim como a dos compostos fenólicos totais não se mostraram alteradas após a competição.

\section{DISCUSSÃO}

Este estudo avaliou o efeito do exercício de longa duração sobre o estresse oxidativo em uma situação real de competição. Através das técnicas utilizadas, não foi possível identificar dano a lipídeos ou a proteínas de membrana após o meio/ronman.

Duas técnicas distintas para medir o dano a lipídeos foram escoIhidas, a quimiluminescência nos eritrócitos e o TBARS no plasma. De acordo com nossos resultados, o trabalho de Oztasan et al. ${ }^{(19)}$ mostrou que ratos submetidos a exercício até a exaustão, após ter recebido um treinamento aeróbio (cinco vezes por semana, ao longo de oito semanas), estavam protegidos do dano a lipídeos, avaliado em eritrócitos através do malondialdeído (MDA), quando comparados com ratos sedentários. Entretanto, os níveis plasmáticos do MDA podem não refletir o real estado do EO. No trabalho de Skenderi et al. ${ }^{(20)}$, utilizando outros marcadores de dano a lipídeos, houve aumento nos níveis de 8-iso-PGF ${ }_{2 a}$ e redução dos níveis de MDA. Os autores justificam a discrepância entre seus resultados indicando o 8-iso-PGF ${ }_{2 a}$ como marcador mais confiável para avaliar a lipoperoxidação.

Ao contrário dos nossos achados, Pétibois e Déléris ${ }^{(5)}$ encontraram dano a lipídios e proteínas. Os autores demonstraram que em indivíduos treinados, após 120 min de exercício com intensidade crescente em cicloergômetro ( 50 a $75 \%$ do $\dot{\text { VO }}{ }_{2 m a ́ x}$ ), os fosfolipídeos da membrana dos eritrócitos sofreram ataque das espécies reativas de oxigênio (ERO), e que a carbonilação verificada pareceu estar ligada aos efeitos combinados do aumento da acidose e da desidratação celular. A técnica de espectrometria FT-IR utilizada foi apresentada como uma ferramenta capaz de caracterizar mudanças estruturais nas moléculas, em especial nos eritrócitos ${ }^{(5)}$. Além disso, níveis elevados de $F_{2}$-isoprostanos durante uma ultramaratona de $50 \mathrm{~km}$ oferecem fortes evidências de que o dano a lipídeos tenha resultado de estresse oxidativo aumentado em resposta ao exercício ${ }^{(4)}$. Após uma ultramaratona de $80 \mathrm{~km}$, os valores de $F_{2}$-isoprostanos aumentaram significativamente, tanto no grupo placebo quanto no grupo suplementado com vitamina $C$, indicando que a corrida induziu o estresse oxidativo(21).

Mastaloudis et al. ${ }^{(22)}$ também encontraram aumento na lipoperoxidação medida através dos $F_{2}$-isoprostanos plasmáticos, após uma ultramaratona de $50 \mathrm{~km}$. Esse resultado foi observado somente no grupo não suplementado, o grupo que recebeu suplementação de vitaminas C e E foi protegido. Outro achado interessante do mesmo estudo foi que a resposta inflamatória induzida pelo exercício não foi influenciada pela suplementação de antioxidantes. Essa resposta inflamatória é importante, pois estimula a recuperação do exercício por induzir a regeneração do tecido danificado e o recrutamento da proliferação de células satélite. Isso é relevante, pois a prevenção da inflamação poderia inibir a adaptação muscular à atividade física, o chamado efeito do treinamento físico.

Em nosso estudo não foi observada alteração no dano a proteínas após a competição. Da mesma forma, Miyazaki et al. ${ }^{(23)}$ não observaram mudança nos marcadores de oxidação protéica, tanto após 12 semanas de treinamento quanto após exercício exaustivo, porém, avaliando sujeitos saudáveis, não atletas. Em outro modelo de exercício, Sentürk et al. ${ }^{(24)}$, avaliando atletas em um teste incremental máximo, observaram dano às proteínas. Os atletas em nosso estudo não necessariamente chegaram à exaustão, tinham experiência de treinamento maior que 12 semanas e provavelmente mantiveram-se no metabolismo aeróbio a maior parte da competição, o que poderia explicar a diferença entre os resultados encontrados. Ainda assim, Radák et al.(25) observaram, após $93 \mathrm{~km}$ de corrida, aumento nas carbonilas plasmáticas e que esse aumento estava correlacionado com as carbonilas urinárias, sugerindo um marcador não invasivo de EO.

Quanto aos aspectos antioxidantes, as propriedades não enzimáticas do ácido úrico conferem efeitos scavenger sobre os radicais livres in vivo, o que foi demonstrado no estudo de Waring et al. ${ }^{(26)}$, pela administração de ácido úrico, o qual temporariamente aumentou a concentração circulante do ácido úrico e reduziu o EO induzido pelo exercício em indivíduos jovens e saudáveis. Em nosso estudo, observamos aumento de aproximadamente 48\% nas concentrações plasmáticas de ácido úrico após a competição de meio Ironman, de acordo com os resultados de Mastaloudis et al. ${ }^{(4)}$ em 2001, onde os níveis de acido úrico também aumentaram durante uma ultramaratona de $50 \mathrm{~km}$. Mastaloudis et al.(22) em 2004, encontraram aumento do ácido úrico após uma corrida de ultramaratona, os quais se mantiveram elevados mesmo dois dias após a corrida. Essa elevação pode ser explicada por aumento da oxidação da purina com o exercício. As necessidades energéticas aumentadas, características do exercício vigoroso, regulam várias vias metabólicas, incluindo a adenilato ciclase (ou mioquinase no músculo). Essa enzima é responsável pela produção de um ATP e um AMP a partir de dois ADP. Enquanto o ATP é usado para energia, o AMP é degradado a ácido úrico.

No estudo de Ginsburg et al. ${ }^{(7)}$, os autores observaram em triatletas que realizaram o Ironman do Havaí, uma proteção aos lipídeos de membrana. Dentre as possíveis explicações para essa proteção citam: (a) maior liberação de estradiol no plasma, (b) indução dos antioxidantes que ocorrem naturalmente no plasma ou (c) redução dos pró-oxidantes no plasma. Sendo assim, podemos sugerir que, em nosso estudo, o ácido úrico, um dos antioxidantes plasmáticos, estando aumentado após o meio Ironman. poderia ser um dos responsáveis por esse efeito protetor.

Em relação ao sistema de defesa antioxidante enzimático, a atividade da enzima catalase nos eritrócitos não sofreu alteração significativa 
após o meio Ironman, o que está de acordo com o estudo de Marzatico et al.(27), avaliando uma meiamaratona. Por outro lado, no estudo de Aguilo et al. ${ }^{(28)}$, ciclistas profissionais realizaram um exercício exaustivo com percurso em montanha de $171 \mathrm{~km}$; após o final da prova, a atividade da enzima catalase mostrou-se aumentada em torno de 30\%, voltando aos valores basais após $3 \mathrm{~h}$ de recuperação. Apesar do meio Ironman ser um exercício de longa duração, não necessariamente foi considerado exaustivo aos atletas, assim como o percurso realizado era plano, e talvez por isso não tenhamos observado alteração na atividade da CAT.

Além disso, observamos redução de aproximadamente 10\% na atividade da enzima superóxido dismutase após o meio Ironman (tabela 1). Esse resultado é diferente do encontrado por outros autores que estudaram protocolos diferenciados de exercício, como Palazzetti et al.(29), avaliando triatletas submetidos a um duatlo após protocolo de supertreinamento, e Aguilo et al. ${ }^{(28)}$, avaliando ciclistas profissionais após uma prova exaustiva em montanha de $171 \mathrm{~km}$, onde a atividade da SOD não se modificou após a competição. Já Oztasan et al. ${ }^{(19)}$ verificaram que, após o exercício exaustivo agudo, a atividade da enzima CuZn-SOD nos eritrócitos de ratos treinados estava aumentada.

Podemos observar que os dados da literatura são conflitantes, já que apresentam redução, aumento ou ausência de mudança nos parâmetros de EO pós-exercício agudo. A discrepância dos resultados entre os estudos pode resultar de diferentes técnicas de medida, tipos de cobaia, esporte e treinamento, assim como o modelo de exercício agudo realizado. Além disso, as diferenças entre o resultado dos estudos podem ser atribuídas ao balanço inicial de enzimas antioxidantes, pois, conforme Tauler et al. (30), o tipo de ERO gerada nos eritrócitos é influenciado por diversos fatores, incluindo a intensidade do exercício e o balanço das enzimas antioxidantes antes do exercício.

Sentürk et al. ${ }^{(24)}$ avaliaram os eritrócitos de indivíduos sedentários e atletas após um protocolo exaustivo em bicicleta, tanto antes quanto após um período de dois meses de suplementação com as vitaminas A, C e E. Os autores demonstraram que uma simples sessão de exercício intenso foi capaz de aumentar os parâmetros de estresse oxidativo e in-

\section{REFERÊNCIAS BIBLIOGRÁFICAS}

1. Ji LL. Antioxidants and oxidative stress in exercise. Proc Soc Exp Biol Med. 1999;222(3):283-92. Review.

2. Viña J, Gomez-Cabrera MC, Lloret A, Marquez R, Miñana JB, Pallardó FV, et al. Free radicals in exhaustive physical exercise: mechanism of production, and protection by antioxidants. IUBMB Life. 2000;50(4-5):271-7. Review.

3. Powers SK, Ji LL, Leeuwenburgh C. Exercise training-induced alterations in skeletal muscle antioxidant capacity: a brief review. Med Sci Sports Exerc. 1999;31(7):987-97. Review.

4. Mastaloudis A, Leonard SW, Traber MG. Oxidative stress in athletes during extreme endurance exercise. Free Radic Biol Med. 2001;31(7):911-22.

5. Pétibois $C$, Déléris $G$. Evidence that erythrocytes are highly susceptible to exercise oxidative stress: FT-IR spectrometric studies at the molecular level. Cell Biol Int. 2005;29(8):709-16.

6. Niess AM, Baumann M, Roecker K, Horstmann T, Mayer F, Dickhuth HH. Effects of intensive endurance exercise on DNA damage in leucocytes. J Sports Med Phys Fitness. 1998;38(2):111-5.

7. Ginsburg GS, O'Toole M, Rimm E, Douglas PS, Rifai N. Gender differences in exercise-induced changes in sex hormone levels and lipid peroxidation in athletes participating in the Hawaii Ironman triathlon. Ginsburg-gender and exercise-induced lipid peroxidation. Clin Chim Acta. 2001;305(1-2):131-9.

8. McAnulty SR, McAnulty LS, Nieman DC, Morrow JD, Shooter LA, Holmes S, et al. Effect of alpha-tocopherol supplementation on plasma homocysteine and oxidative stress in highly trained athletes before and after exhaustive exercise. J Nutr Biochem. 2005;16(9):530-7.

9. Knez WL, Jenkins DG, Coombes JS. Oxidative stress in half and full Ironman triathletes. Med Sci Sports Exerc. 2007;39(2):283-8.

10. Marklund SL. Direct assay with potassium superoxide. In: Greenwald R, editor. Handbook of methods for oxygen radical research. Boca Raton, FL: CRC Press, 1985;249-55.

11. Boveris A, Chance B. The mitochondrial generation of hydrogen peroxide. General properties and effect of hyperbaric oxygen. Biochem J. 1973;134(3):707-16.

12. Flohe L, Gunzler WA. Assays of glutathione peroxidase. Methods Enzymol. 1984;105:114-21.

13. Gonzalez Flecha B, Llesuy S, Boveris A. Hydroperoxide-initiated chemiluminescence: an assay for oxidative stress in biopsies of heart, liver, and muscle. Free Radic Biol Med. 1991;10(2):93-100.

14. Llesuy SF, Milei J, Gonzalez Flecha BS, Boveris A. Myocardial damage induced by doxorubicins: hydroperoxide-initiated chemiluminescence and morphology. Free Radic Biol Med. 1990;8(3):259-64.

15. Draper HH, Hadley M. Malondialdehyde determination as index of lipid peroxidation. Methods Enzymol. 1990;186:421-31 duzir deterioração da função e estrutura dos eritrócitos em sedentários, o que foi prevenido por dois meses de suplementação antioxidante. Contudo, esse comportamento não se repetiu nos indivíduos que eram treinados. Este estudo também mostrou que a diferença entre os eritrócitos sedentários e treinados pode ser parcialmente dependente da jovem população de eritrócitos presente nos indivíduos treinados, os quais são mais resistentes ao estresse oxidativo. Em resumo, o exercício afeta as propriedades dos eritrócitos e leva à hemólise por um mecanismo oxidativo em sedentários, mas não em treinados. O estresse oxidativo pode ser um importante fator responsável pela destruição dos eritrócitos observada após treinamento vigoroso, agudo em sedentários e/ou em início do período de treinamento. No estudo atual esses parâmetros referentes aos eritrócitos não foram mensurados, mas em estudos futuros esse tipo de informação deve ser incluído para melhorar o entendimento sobre a questão do estresse oxidativo.

Podemos indicar ainda como limitação do estudo o fato de a alimentação, hidratação e suplementação durante a competição não terem sido controladas. Seria interessante em uma próxima intervenção avaliar maior número de atletas, incluindo a medida da enzima creatina quinase, marcadora de dano muscular, e acompanhar o estresse oxidativo durante o período de recuperação após a competição.

\section{CONCLUSÃO}

Após a competição de meio Ironman foi observado aumento nas concentrações plasmáticas de ácido úrico e redução na atividade da enzima superóxido dismutase. Os parâmetros de dano a lipídeos e proteínas de membrana não se alteraram, assim como não houve mudança na atividade das enzimas catalase e glutationa peroxidase. Sendo assim, podemos sugerir que esse grupo de atletas, após uma competição de longa duração, não sofreu estresse oxidativo, possivelmente devido à liberação de acido úrico e outros antioxidantes no plasma.

Todos os autores declararam não haver qualquer potencial conflito de interesses referente a este artigo.

16. Reznick AZ, Packer L. Oxidative damage to proteins: spectrophotometric method for carbonyl assay. Methods Enzymol. 1994;233:357-63.

17. Waterman P, editor. MS. Analysis of phenolic plant metabolites. London: Blackwell Scientific Pubs, 1994.

18. Lowry OH, Rosebrough NJ, Farr AL, Randall RJ. Protein measurement with the Folin phenol reagent. J Biol Chem. 1951;193(1):265-75.

19. Oztasan N, Taysi S, Gumustekin K, Altinkaynak K, Aktas O, Timur H, et al. Endurance training attenuates exercise-induced oxidative stress in erythrocytes in rat. Eur J Appl Physiol. 2004;91(5-6):622-7.

20. Skenderi KP, Tsironi M, Lazaropoulou C, Anastasiou CA, Matalas AL, Kanavaki I, et al. Changes in free radical generation and antioxidant capacity during ultramarathon foot race. Eur J Clin Invest. 2008;38(3):159-65.

21. Nieman DC, Henson DA, McAnulty SR, McAnulty L, Swick NS, Utter AC, et al. Influence of vitamin C supplementation on oxidative and immune changes after an ultramarathon. J Appl Physiol. 2002;92(5):1970-7.

22. Mastaloudis A, Morrow JD, Hopkins DW, Devaraj S, Traber MG. Antioxidant supplementation prevents exercise-induced lipid peroxidation, but not inflammation, in ultramarathon runners. Free Radic Biol Med. 2004;36(10):1329-41

23. Miyazaki H, Oh-ishi S, Ookawara T, Kizaki T, Toshinai K, Ha S, et al. Strenuous endurance training in humans reduces oxidative stress following exhausting exercise. Eur J Appl Physiol. 2001;84(1-2):1-6.

24. Sentürk UK, Gündüz F, Kuru O, Koçer G, Ozkaya YG, Yesilkaya A, et al. Exercise-induced oxidative stress leads hemolysis in sedentary but not trained humans. J Appl Physiol. 2005;99(4):1434-41.

25. Radák Z, Ogonovszky H, Dubecz J, Pavlik G, Sasvari M, Pucsok J, et al. Super-marathon race increases serum and urinary nitrotyrosine and carbonyl levels. Eur J Clin Invest. 2003;33(8):726-30.

26. Waring WS, Convery A, Mishra V, Shenkin A, Webb DJ, Maxwell SR. Uric acid reduces exercise-induced oxidative stress in healthy adults. Clin Sci (Lond). 2003;105(4):425-30.

27. Marzatico F, Pansarasa O, Bertorelli L, Somenzini L, Della Valle G. Blood free radical antioxidant enzymes and lipid peroxides following long-distance and lactacidemic performances in highly trained aerobic and sprint athletes. J Sports Med Phys Fitness. 1997;37(4):235-9.

28. Aguilo A, Tauler P, Fuentespina E, Tur JA, Cordova A, Pons A. Antioxidant response to oxidative stress induced by exhaustive exercise. Physiol Behav. 2005;84(1):1-7.

29. Palazzetti S, Richard MJ, Favier A, Margaritis I. Overloaded training increases exercise-induced oxidative stress and damage. Can J Appl Physiol. 2003;28(4):588-604.

30. Tauler P, Aguilo A, Guix P, Jimenez F, Villa G, Tur JA, et al. Pre-exercise antioxidant enzyme activities determine the antioxidant enzyme erythrocyte response to exercise. J Sports Sci. 2005;23(1):5-13. 